\title{
Spontaneous Cerebrospinal Fluid Leak at the Clivus: Two Case Reports and Literature Review
}

ISSN: 2637-7748

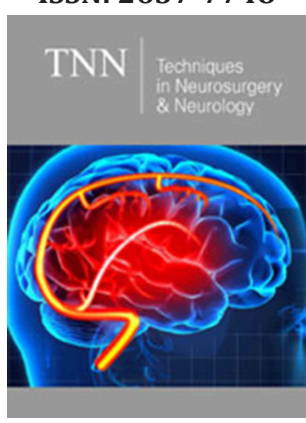

*Corresponding author: Seyed Ali Mousavinejad, Professor of Neurosurgery, Loghman Hakim Hospital, Shahid Beheshti University of Medical Sciences, Iran

Submission: 梅 September 20, 2020

Published: 眥April 21, 2021

Volume 4 - Issue 2

How to cite this article: Mohammad Samadian, Seyed Ali Mousavinejad, Kaveh Ebrahimzadeh, Guive Sharifi, Hesameddin Hoseini Tavassol, et al. Spontaneous Cerebrospinal Fluid Leak at the Clivus: Two Case Reports and Literature Review. Tech Neurosurg Neurol. 4(2). TNN. 000583. 2021

DOI: 10.31031/TNN.2021.04.000583

Copyright@ Seyed Ali Mousavinejad, This article is distributed under the terms of the Creative Commons Attribution 4.0 International License, which permits unrestricted use and redistribution provided that the original author and source are credited.
Mohammad Samadian', Seyed Ali Mousavinejad1*, Kaveh Ebrahimzadeh ${ }^{1}$, Guive Sharifi ${ }^{1}$, Hesameddin Hoseini Tavassol ${ }^{2}$, Kristen Almagro ${ }^{1}$, Omidvar Rezaei $^{2}$ and Hamid Borghei Razavi ${ }^{2}$

${ }^{1}$ Professor of Neurosurgery, Loghman Hakim Hospital, Shahid Beheshti University of Medical Sciences, Iran

${ }^{2}$ Department of Neurosurgery, Pauline Braathen Neurological Center, Cleveland Clinic Florida, USA

\begin{abstract}
Spontaneous or non-traumatic cerebrospinal fluid leaks comprise 5-10\% of all CSF rhinorrhea. Generally, CSF rhinorrhea occur at Cribriform plate, sella, sphenoid sinus and ethmoid air. Primary CSF rhinorrhea from clival defect is extremely rare. We herein describe two cases of spontaneous CSF rhinorrhea through the clivus repaired with endoscopic endonasal trans sphenoid approach. Moreover, we collected evidence in the literature regarding potential etiology, symptom and treatment (which occurred in the case reported).
\end{abstract}

\section{Introduction}

Spontaneous or non-traumatic cerebrospinal fluid leaks comprise $5 \%-10 \%$ of all Cerebrospinal Fluid (CSF) rhinorrhea [1,2]. Generally, CSF rhinorrhea can occur at the cribriform plate, sella, sphenoid sinus, or ethmoid air cells $[3,4]$. However, primary CSF rhinorrhea due to clival defect is extremely rare. The current study describes two cases of spontaneous CSF rhinorrhea through the clivus which were repaired with endoscopic endonasal trans-sphenoidal approach. Such cases are extremely rare and upon literature review, only 16 cases with clival defect are reported thus far. The peculiar aspect of this case report is related to its rarity. Moreover, evidence was collected from the literature regarding potential etiology, symptom, and treatment (occurred in the reported cases).

\section{Case 1}

A 36 -year-old female was admitted for three weeks of clear watery discharge from the right nostril, which was aggravated in prone position. The patient denied any recent trauma. A review of systems was negative except for headaches and nasal discharge. The nasal fluid tested positive for beta- 2 transferrin, indicating that the fluid was CSF. Brain MRI revealed that the sphenoid sinus was filled with Cerebrospinal Fluid (CSF) and sagittal T2 weighted MRI revealed a fistula tract from prepontine cistern to sphenoid sinus (Figure 1). There was no evidence of benign intracranial hypertension. Computed tomography cisternography revealed that the contrast material passed from the prepontine cistern into the sphenoid sinus through this bone defect in the clivus (Figure 1). Before surgery, a lumbar puncture was performed to administer $0.25 \mathrm{~mL}$ of $10 \%$ fluorescein with $10 \mathrm{~mL}$ of Cerebrospinal Fluid (CSF) to help visualize CSF leaks during surgery and to ensure there was no leak after reconstruction of the defect. The patient underwent endoscopic trans nasal transsphenoidal surgery. The anterior and middle portions of the clivus were exposed between both carotid arteries. During surgery, the defect was defined to the left of the midline in the clivus. The basilar artery was seen through the defect in prepontine cistern (Figure 2). The defect was closed with a multilayer reconstruction consisting of fat, fascia lata, and naso septal flap (Figure 3). There was no recurrence of CSF leak at 2 years follow-up. 


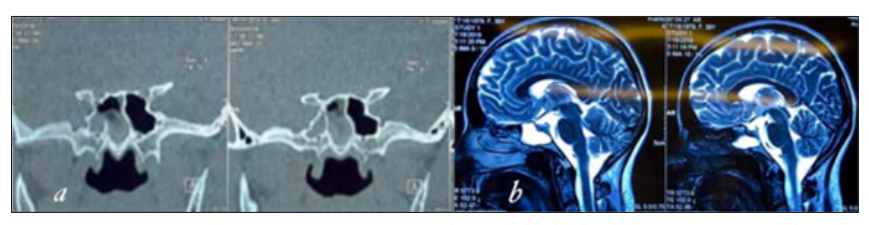

Figure 1: (a) Coronal CT cisternography sphenoid sinus filled with CSF. (b) Sagittal T2-weighted MRI of the brain showing cerebrospinal fluid (CSF) leak into the sphenoid sinus trough clival defect.

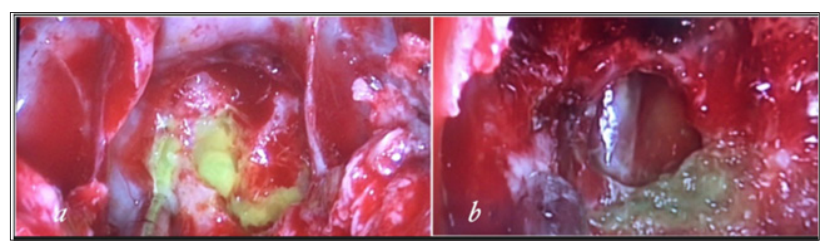

Figure 2: (a) Intraoperative endoscopic view show CSF leakage from clivus defect just inferior to sella .(b) basilar artery in prepontine cistern behind the defect.

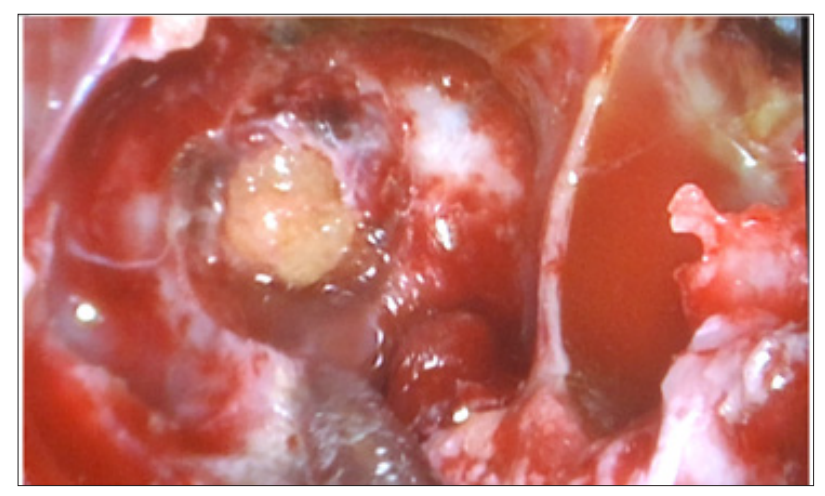

Figure 3: Endoscopic view pos repair with abdominal fat.

\section{Case 2}

A 57-year-old man referred for clear watery discharge from the right nostril of no obvious cause. He suffered from intermittent rhinorrhea starting 6 months prior to arrival. He reported recent history of bacterial meningitis one month ago, which was treated successfully at an outside hospital. On admission, he had no focal neurological deficits. Nasal fluid tested positive for beta2 transferrin. Brain MRI revealed that the right sphenoid sinus was filled with CSF (Figure 4). CT cisternography showed that the contrast material passed from the prepontine cistern into the sphenoid sinus through this bone defect in the clivus. (Figure 4). After intrathecal administration of $0.25 \mathrm{~mL}$ of $10 \%$ fluorescein with $10 \mathrm{~mL}$ of cerebrospinal fluid the patient underwent endoscopic trans nasal approach. After stripping the mucosa from posterior wall of sphenoid sinus, CSF leak was observed in the upper region of clivus just below the sella at the midline (Figure 5). The defect was closed by abdominal fat and reinforced by fascia lata and naso septal flap. At the During 30-month follow-up appointment, no signs of recurrence were found. In both of the above reviewed cases, a lumbar drain was not placed pre or postoperatively.

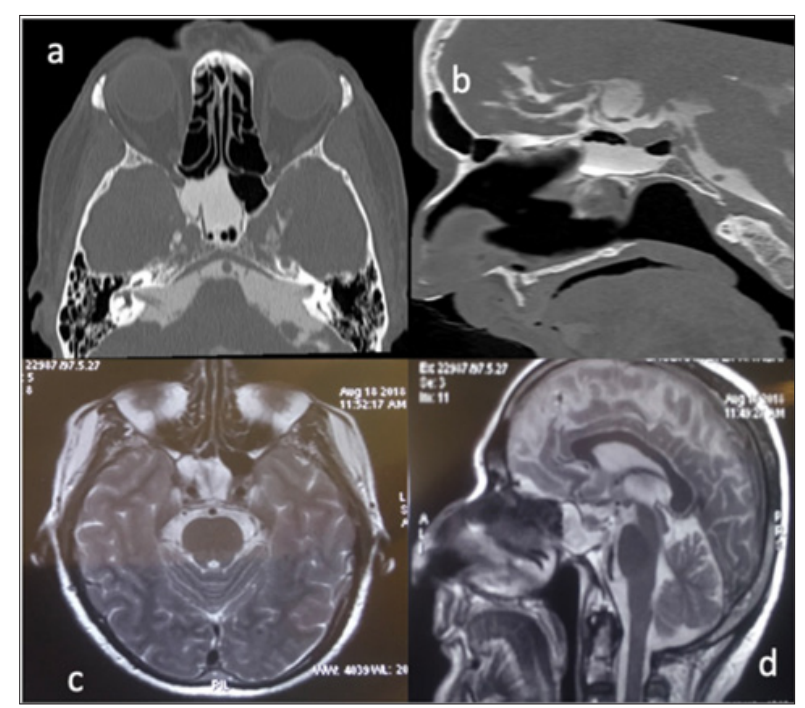

Figure 4: (a) CT Cisternography showing right sphenoid sinus filled with CSF and (b) the entry of cerebrospinal fluid into the sphenoid sinus. (c) axial T2 weight MRI revealed right sphenoid sinus filled with CSF (d) CSF leakage from prepontine cistern to sphenoid sinus.

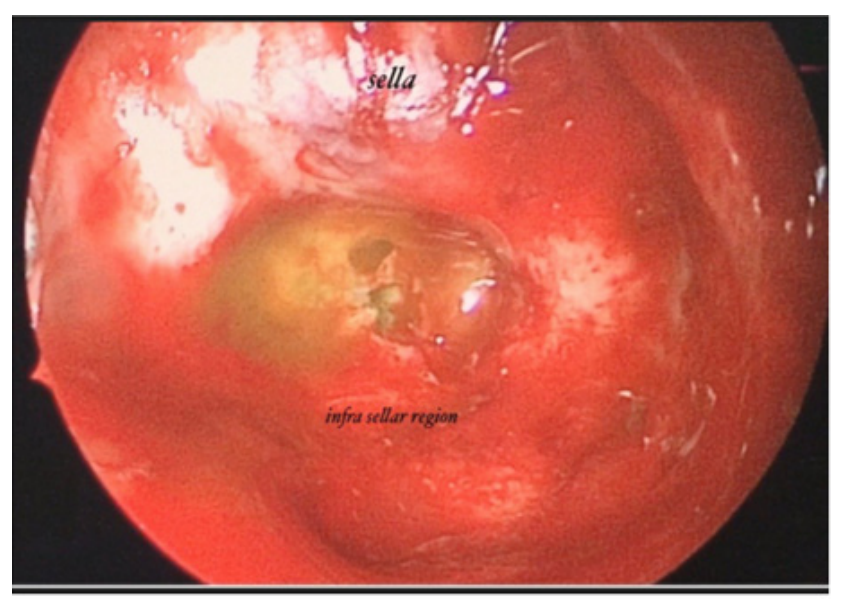

Figure 5: Intraoperative endoscopic view show CSF leakage from clivus defec.

\section{Discussion}

Cerebrospinal Fluid (CSF) leaks most commonly result from nonsurgical trauma (80\%-90\% of cases), followed by surgical procedures $(16 \%)$, and nontraumatic or spontaneous causes $(4 \%)[5,6]$. first subcategorized spontaneous CSF rhinorrhea in two groups in 1964; primary spontaneous, when there is no cause for skull defect, and secondary spontaneous, when a cause can be found $[6,7]$. Defects in the roof of the ethmoid sinus or in the floor of the anterior cranial fossa contribute to the most common site of fistula in the patient with traumatic CSF rhinorrhea. However, for primary spontaneous CSF fistulas, a sphenoidal fistula is most common $(60 \%)$. In these cases, the junction of the floor of the middle cranial fossa to the lateral wall of the sphenoid sinus is the most common site of CSF leak through sphenoid sinus [8], however, primary spontaneous CSF leaks from clival defect are extremely 
rare. In a study by Hooper [9] on 138 sphenoid bones, the defect of bones connecting the sphenoid to the cranium leading to CSF leak was observed in $5 \%$ of cases; all of which were in lateral wall of the sphenoid sinus $[9,10]$. To the best of the authors` knowledge, only 16 cases of spontaneous CSF leaks from clival defect are reported thus far. The exact pathophysiology of CSF clival fistula is debated. Morley [11] postulated that congenital bone defects in the middle fossa can explain the leaks through the lateral extensions of the sphenoid sinus [11]. However, from anatomic point of view, there is no clear embryological evidence to explain clival defect causing CSF rhinorrhea. The clivus is a bony structure composed of the fusion of the posterior portion of the sphenoid body (basisphenoid) and the basilar part of the occipital bone (basioccipital) at the sphenooccipital synchondrosis (SOS). SOS fusion can occur at any age, but it usually happens before adolescence. SOS may persist into adult life and may be mistaken for a fracture or defect. However, this synchondrosis is caudal to the future sphenoid sinus; otherwise, clival ossification would be a continuous enchorial ossification without fusion point that could explain bone defect $[12,13]$. According to Faizuddin Ahmad [7] most authors believe that excessive pneumatization of sphenoid sinus causes a thin bony wall at some points of clivus and sphenoid. These phenomena, combined with other potential factors such as arterial pulsation and continuous CSF pressure wave ultimately lead to the bone defect at the clivus and CSF leak $[7,14,15]$. Sixteen cases of spontaneous CSF leak at the clivus were reported from 1995 to 2018. (Table 1) In all patients, the defect was localized in the upper clivus. In all sixteen cases reported thus far CSF leak from clival defect occurred in adult patients, which may explain the role of CSF pressure pulsation as a predisposing factor. The CSF pressure pulsation reaches its maximum point in adults, approximately three times higher than that of infants. Eleven of the sixteen patients were female. In 1995, Coitero reported the first two cases of CSF fistula through the clivus. In one case they suggested that basilar artery pulsations over a thinned bone structure could be the cause of clival defect [16]. Radiographic features of increased intracranial pressure (ICP) such as empty sella syndrome (80\%) and arachnoid pits (63\%) are often observed in patients with spontaneous CSF rhinorrhea. However, in a case report by Thibaut van Zele in spontaneous clival CSF leak, radiologic signs of increased ICP (empty sella and/or arachnoid pits) were observed only in two cases of four patients [17]. In all of the current study patients, the CSF pressure was normal and magnetic resonance findings were not suggestive of benign intracranial hypertension. Radiologic evaluation of CSF leaks is a diagnostic challenge that often involves multiple imaging studies. Various imaging studies such as contrast-enhanced computed tomography (CT) cisternography, radionuclide cisternography, and MR cisternography are employed in the diagnosis of such cases. To date, contrast-enhanced CT cisternography is the standard for CSF leak evaluation with a sensitivity of $76-100 \%$ [18,19]. However, cisternography requires intrathecal injection via lumbar puncture which can cause discomfort for patients. Additionally, CSF leak is often an intermittent phenomenon, therefore the sensitivity of cisternography depends on the timing of the examination. Additional MRI (with T2-weighted sequences) can be helpful if parenchymal or meningeal herniation is suspected [20,21]. Of the above referenced two case studies, one patient's sphenoid sinus was filled with cerebrospinal fluid and defect was discovered in the upper part of the clivus. Retrospectively, in cases suspected of clival defect, the T2-weighted sequence of mid-sagittal MRI showed CSF fistula from prepontine cisterna to sphenoid sinus. Several surgical approaches can be utilized to repair CSF rhinorrhea. However, to date endoscopic transnasal transsphenoid surgery is established as the standard technique to repair CSF leak. This approach is less invasive, with lower morbidity and mortality, excellent view of the surgical field, and a higher success rate [22-25]. In the current study, endoscopic transnasal approach was performed for fistula repair in both patients. All defects were closed with a combination of fat, fascia, and a free or nasoseptal flap. Review of the literature highlighted the fact that identification of the bone/dural defect responsible for CSF leakage was the most important point for successful surgical intervention, since a missed site can lead to improper treatment and recurrence of the leak $[25,26]$. In a report by Vivek Tandon, sphenoid sinus packing was performed when the exact site of the leak was not identified and the patient presented with recurrent rhinorrhea and meningitis [24]. In a report by Luana Antunes Maranha, the patient underwent anterior skull base repair via bifrontal craniotomy three times since the exact site of leakage was not defined and the patient presented with recurrent CSF leak. Finally, the patient underwent endoscopic transnasal approach for clival defect closure [27]. In some cases, there is more than one defect that should be repaired. For example, in a study by Satiawaty Mohindra, a patient had two concomitant defects. In the first surgery, clival defect was missed; the right cribriform plate and right sphenoid sinus defect closures were performed. CSF rhinorrhea recurred after one month and the patient underwent revision surgery. In revision surgery, previous repair site did not show leakage, but a high-pressure leak was observed through posterior wall of sphenoid sinus from clivus [28]. In all of the current study patients, clival defect was the only defect causing CSF leak. To date, various materials such as mucoperichondrium, cartilage, fat, fascia, and fibrin glue have been utilized to seal the fistula with different success rates. Fat and fascia were used for defect closure in all of the patients in the current study, comparable to patients with spontaneous CSF leak or repair of dural defect after adenoma surgery. Similar to Hegazy, we believe that the material used in the closure of the fistula is not important in the success of the intervention. The important key to success is the determination of the bony edge surrounding the defect [29]. Similar to the other authors, we reserved lumbar drainage for patients with elevated intracranial pressure. In both patients, the conservative measures such as bed rest, elevation of the head, and avoidance of straining activities were implemented after surgery [30]. 
Table 1: Published cases of clival defect leading spontaneous CSF leak.

\begin{tabular}{|c|c|c|c|c|c|c|c|}
\hline Case Reference & Sex & Age & Meningitis & $\begin{array}{l}\text { Duration of } \\
\text { CDF Rinorrhea }\end{array}$ & $\begin{array}{l}\text { Previous Surgery with } \\
\text { no Finding Defect }\end{array}$ & $\begin{array}{l}\text { Surgical Approach } \\
\text { for Defect Closure }\end{array}$ & Reccurent \\
\hline Guang Yong Chen [18] & Male & 71 & - & 3week & NO & ETSS & NO \\
\hline Vivek Tandon [24] & Female & 55 & + & 3week & YES [ETSS] & $\begin{array}{l}\text { sublabial rhino sep- } \\
\text { taltranssphenoidal }\end{array}$ & NO \\
\hline Sheikh Asad [10] & Female & 64 & + & $2 y$ & NO & ETSS & YES \\
\hline Satiawaty Mohindra [28] & Female & 35 & - & $2 \mathrm{~m}$ & YES [ETSS] & ETSS & NO \\
\hline Krzysztof Oleś [25] & Female & 60 & + & $3 y$ & NO & ETSS & NO \\
\hline \multirow{6}{*}{ Thibaut Van Zele [17] } & Female & 37 & - & $2 \mathrm{~m}$ & NO & ETSS & NO \\
\hline & Female & 61 & - & $4 \mathrm{~m}$ & NO & ETSS & NO \\
\hline & Female & 78 & - & $3 \mathrm{~m}$ & NO & ETSS & NO \\
\hline & Female & 42 & - & $2 \mathrm{~m}$ & NO & ETSS & NO \\
\hline & Female & 48 & - & $3 \mathrm{~m}$ & NO & ETSS & NO \\
\hline & Male & 50 & - & $1 \mathrm{~m}$ & NO & ETSS & NO \\
\hline Luana Antunes M [27] & Female & 49 & + & $10 y$ & YES [3time] & ETSS & NO \\
\hline \multirow{2}{*}{ Faiz Uddin A [7] } & Male & 52 & + & - & NO & ETSS & NO \\
\hline & Male & 56 & - & $2 y$ & NO & $\begin{array}{l}\text { sublabial rhinoseptal } \\
\text { trans-sphenoidal }\end{array}$ & NO \\
\hline \multirow{2}{*}{ Coiteiro [16] } & Female & 53 & + & $3 \mathrm{mo}$ & NO & ETSS & NO \\
\hline & Male & 43 & + & 1 week & NO & ETSS & NO \\
\hline
\end{tabular}

\section{Conclusion}

Spontaneous CSF rhinorrhea located at the clivus is an extremely rare condition. To date, only 16 cases are reported, and the current study reported a group of two consecutive cases. It seems that a combination of anatomical and functional factors plays a role in the occurrence of this rare phenomenon. To date, endoscopic transnasal approach is the best therapeutic option to repair midline skull base defect such as the current cases. Here, the initial outcome was successful, but long-term follow-up is required.

\section{References}

1. Pérez MA, Bialer OY, Bruce BB, Newman NJ, Biousse V (2013) Primary spontaneous cerebrospinal fluid leaks and idiopathic intracranial hypertension. J Neuroophthal 33(4): 330.

2. Bledsoe JM, Moore EJ, Link MJ (2009) Refractory cerebrospinal fluid rhinorrhea secondary to occult superior vena cava syndrome and benign intracranial hypertension: diagnosis and management. Skull Base 19(04): 279-285.

3. Lopatin AS, Kapitanov DN, Potapov AA (2003) Endonasal endoscopic repair of spontaneous cerebrospinal fluid leaks. Arch Otolaryngol Head Neck Surg 129(8): 859-863.

4. Hubbard JL, McDonald TJ, Pearson BW, Laws ER (1985) Spontaneous cerebrospinal fluid rhinorrhea: evolving concepts in diagnosis and surgical management based on the Mayo Clinic experience from 1970 through 1981. Neurosurgery 16(3): 314-221.

5. Psaltis AJ, Schlosser RJ, Banks CA, Yawn J, Soler ZM (2012) A systematic review of the endoscopic repair of cerebrospinal fluid leaks. Otolaryngol Head Neck Surg 147(2): 196-203.
6. Sharifi G, Mousavinejad SA, Bahrami MH, Eftekharian A, Samadian M, et al. (2019) Delay posttraumatic paradoxical cerebrospinal fluid leak with recurrent meningitis. Asian J Neurosurg 14(3): 964.

7. Ahmad FU, Sharma BS, Garg A, Chandra PS (2008) Primary spontaneous CSF rhinorrhea through the clivus: possible etiopathology. Journal of Clinical Neuroscience 15(11): 1304-1308.

8. Oconnell JE (1964) Primary spontaneous cerebrospinal fluid rhinorrhoea. J Neurol Neurosurg Psychiatry 27(3): 241.

9. Hooper A (1971) Sphenoidal defects a possible cause of cerebrospinal fluid rhinorrhoea. Journal of Neurology, Neurosurgery \& Psychiatry 34(6): 739-742.

10. Asad S, Peters Willke J, Brennan W, Asad S (2017) Clival defect with primary CSF rhinorrhea: a very rare presentation with challenging management. World Neurosurg 106: 1052.

11. Morley T, Wortzman G (1965) The importance of the lateral extensions of the sphenoidal sinus in post-traumatic cerebrospinal rhinorrhoea and meningitis: clinical and radiological aspects. J Neurosurg 22(4): 326-332.

12. Shetty PG, Shroff MM, Fatterpekar GM, Sahani DV, Kirtane MV (2000) A retrospective analysis of spontaneous sphenoid sinus fistula: MR and CT findings. AJNR 21(2): 337-342.

13. Calcaterra TC (1980) Extracranial surgical repair of cerebrospinal rhinorrhea. Ann Otol Rhinol Laryngol 89(2): 108-116.

14. Haetinger RG, Navarro JA, Liberti EA (2006) Basilar expansion of the human sphenoidal sinus: an integrated anatomical and computerized tomography study. Eur Radiol 16(9): 2092.

15. Coiteiro D, Távora L, Antunes JL (1995) Spontaneous cerebrospinal fluid fistula through the clivus: report of two cases. Neurosurgery 37(4): 826828 
16. Van Zele T, Kitice A, Vellutini E, Balsalobre L, Stamm A (2013) Primary spontaneous cerebrospinal fluid leaks located at the clivus. Allergy Rhinol 4(2): 0053.

17. Chen GY, Ma L, Xu ML, Zhang JN, He ZD, et al. (2018) Spontaneous cerebrospinal fluid rhinorrhea: A case report and analysis. Medicine 97(5).

18. Reddy M, Baugnon K (2017) Imaging of cerebrospinal fluid rhinorrhea and otorrhea. Radiol Clinic 55(1): 167-187.

19. Algin O, Hakyemez B, Gokalp G, Ozcan T, Korfali E (2010) The contribution of 3D-CISS and contrast-enhanced MR cisternography in detecting cerebrospinal fluid leak in patients with rhinorrhoea. $\mathrm{Br} \mathrm{J}$ Radiol 83(987): 225-232.

20. Hegarty S, Millar J (1997) MRI in the localization of CSF fistulae: is it of any value? Clinical Radiology 52(10): 768-770.

21. Muscatello L, Lenzi R, Dallan I, Seccia V, Marchetti M (2010) Endoscopic transnasal management of cerebrospinal fluid leaks of the sphenoid sinus. J Cranio Maxillof Surg 38(5): 396-402.

22. Elrahman HA, Malinvaud D, Bonfils NA, Daoud R, Mimoun (2009) Endoscopic management of idiopathic spontaneous skull base fistula through the clivus. Arch Otolaryngol Head \& Neck Surg 135(3): 311-315.

23. Tandon V, Garg K, Suri A, Garg A (2017) Clival defect causing primary spontaneous rhinorrhea. Asian J Neurosurg 12(2): 328.
24. Oleś K, Składzien J, Betlej M, Chrzan R, Mika J (2015) Spontaneous cerebrospinal fluid leak at the clivus. Case Report 10(4): 593.

25.Virk JS, Elmiyeh B, Saleh HA (2013) Endoscopic management of cerebrospinal fluid rhinorrhea: The charing cross experience. J Neurolog Surg Part B: Skull Base 74(02): 061-067.

26. Maranha LA, Corredato RDA, Araújo JC (2012) Nontraumatic clival cerebrospinal fluid rhinorrhea. Arquivos de neuro-psiquiatria 70(7): 550-551.

27. Mohindra S, Mohindra S, Joshi dk, Sodhi HB (2017) Delayed spontaneous cerebrospinal leak through clival recess: emphasis on technique of repair. Clinical Rhinology an International Journal 10: 42-44.

28. Hegazy HM, Carrau RL, Snyderman CH, Kassam A, Zweig (2000) Transnasal endoscopic repair of cerebrospinal fluid rhinorrhea: A metaanalysis. The Laryngoscope 110(7): 1166-1172.

29. Casiano RR, Jassir D (1999) Endoscopic cerebrospinal fluid rhinorrhea repair: is a lumbar drain necessary? Otolaryngol Head Neck Surg 121(6): 745-750.

30. Yadav YR, Parihar V, Janakiram N, Pande S, Bajaj J (2016) Endoscopic management of cerebrospinal fluid rhinorrhea. Asian J Neurosurg 11(3): 183. 